\title{
A collaborative transportation proposal for urban deliveries: costs and environmental savings
}

\author{
D. Tacla, O. F. Lima Jr \& R. C. Botter \\ Departamento de Engenharia Naval e Oceânica, \\ Universidade Estadual de São Paulo, Brazil
}

\begin{abstract}
What is the best way to make deliveries in urban areas: with big trucks or small cargo vehicles? Which one is better if we think about environmental problems and traffic? Just one medium-sized truck can substitute fifteen small vehicles. A Collaborative transportation plan and operation between shippers, carriers and customers could solve this problem, but only a complete collaboration could do it. Fifteen small vehicles need fifteen different time slots to unload, with fifteen drivers and fifteen engines throwing out smoke and money in the air. This paper presents a proposal to plan collaborative transportation between big players (carrier, shippers and customers) to optimize transportation using trucks with cargo consolidated between various shippers going to one destination (a supermarket for example). The methodology used is a combination between collaborative planning (CPFR) and a mathematical model to optimize the fleet. This study brings the CPFR concept, in a practical approach, to urban deliveries, trying not to contribute just another case for optimization, but to lesson the environmental and traffic impacts on highly populated cities. The paper also presents a case study creating a great opportunity for the development of a methodology capable of contributing to the framing of collaborative transportation, as well as to alternatives, which mitigate issues caused by metropolitan cargo transportation. The case study is a real case concerning one of the biggest retail companies in the world, the Distribution Center in Brasilia (BDC), Brazil. The database has 18,314 lines with cargoes, trucks, information of receipt, goods and invoices.
\end{abstract}

Keywords: collaborative transportation; cargo transportation, urban delivery. 


\section{Introduction}

The Urban Cargo Transportation has became more and more important because it impacts directly on the lives of the people and traffic in the central regions (e.g. Lima Jr. [4]). This issue balances Thirds Part Logistics with productivity; the normal people who have to live with the trucks and their noise and pollution; the governments that try to fix the situation with laws and rules without harming the economy and businesses. This is a difficult situation which only gets worse.

Regarding Collaborative Transportation, several authors have viewed the current stage of logistics as the 'new wave', and "Collaborative Logistics". It has been so named by Beau Browning and Andrew White, both members of the CTM Committee.

Collaborative transportation is one aspect, but maybe the most important one, of Collaborative Logistics; this would be the evolution of the most recent stage (e.g. Bowersox [1]), called Supply Chain Management. As transportation is the highest resource-consuming process in logistics, it must be approached with due relevance. Collaborative Transportation emerges naturally from this scenario.

Collaborative Transportation takes place, conceptually, when one can use, or share, the same transportation facilities for a closed cycle of moving cargo. One would need to gather the participants of the same logistics chain, or shippers that might offer complementary cargo - such as cargo compatible with the transportation facilities available on the complimentary route - thus securing the return cargo. The Urban Cargo transportation in metropolitan areas can be analyzed with a different focus: Third Party Logistics (or the carrier) impacted by traffic, problems with access and long queues to unload; cargo transportation users impacted by deliveries delays and freight costs; people are also impacted by things like heavy traffic and pollution (noise and smoke). On the other hand, the Urban Cargo transportation is hugely important for the economy on a global scale but also to local businesses.

The question raised here is: how do we put this concept of Collaborative Transportation into practice to mitigate Urban Cargo Transportation issues? If specialized literature shows a clear tendency pointing to "Collaborative Logistics" as the new phase in logistics, and collaborative transportation as a new important tool in obtaining results, its actual application calls for an objective project. The case studied in this paper and the databases available offer great opportunities for the development of a methodology capable of contributing to the instigation of Collaborative Transportation, as well as alternatives to reduce Urban Cargo issues. In this way, it becomes technically possible to transport more cargo, from more than one shipper in the same vehicle, on the return journey. When this does not happen, the cargo must go to its destination through much more, smaller vehicles. The shippers and buyers should work together with carriers; if they do, all players in this game will win: there will be lower costs involved in the logistical system, better life for third parties, with fewer lines and more productivity, and the community gains by incurring less pollution. This is the approach taken by this research. 


\section{Characterization of cargo urban transportation in metro areas}

The basic way for the system to work (e.g. Desrochers [3]), regarding deliveries in metropolitan areas or urban areas, is through consolidated cargoes to microregions by the carriers. The retail companies (focus on retails companies) normally do not pay the freight cost, they buy the products from the suppliers to put in their stores or distribution centres (DC); therefore the suppliers hire transportation companies to pick up the goods and deliver them to the retailers. The main agent of cargo consolidation is the carrier. The carrier picks up the cargo from the supplier and moves this cargo to his site; in this area he delivers cargoes to a macro-region and transfers them to a local site (delivery region). This cargo is then delivered to the final customer (the retailer) in smaller trucks. Figure 1 shows those operations in a schematic way with more details. What are the issues and consequences stemming from this form of operation? If on one hand this is the classical way and maybe more feasible from a commercial point of view; on the other hand it is a bad way from the point of view of the logistic and optimization focus. Some aspects justifying this are indicated in Table 1:

Table 1: $\quad$ Issues and losses - classical delivery.

\begin{tabular}{|l|l|l|}
\hline Issue & Operational or Financial Losses & Urban population losses \\
\hline $\begin{array}{l}\text { Smaller trucks than } \\
\text { big trucks or FTL. }\end{array}$ & $\begin{array}{l}\text { Transportation system losses } \\
\text { indirectly increase costs and } \\
\text { decrease productivity. }\end{array}$ & $\begin{array}{l}\text { More vehicles circulating and } \\
\text { blocking the traffic. More sound } \\
\text { and air pollution. }\end{array}$ \\
\hline More unloading. & $\begin{array}{l}\text { Logistic system losses with directly } \\
\text { increased costs and low } \\
\text { productivity. }\end{array}$ & $\begin{array}{l}\text { More vehicles moving inside } \\
\text { stores or DC areas generating more } \\
\text { sound and air pollution. }\end{array}$ \\
\hline $\begin{array}{l}\text { More queues to } \\
\text { unload trucks. }\end{array}$ & $\begin{array}{l}\text { Logistic system losses indirectly } \\
\text { increase costs and decrease } \\
\text { productivity. }\end{array}$ & $\begin{array}{l}\text { More vehicles moving inside } \\
\text { stores or DC areas generating more } \\
\text { sound and air pollution. }\end{array}$ \\
\hline $\begin{array}{l}\text { Delays caused by } \\
\text { queues on first stop. }\end{array}$ & $\begin{array}{l}\text { Logistic system losses indirectly } \\
\text { increase costs. }\end{array}$ & $\begin{array}{l}\text { Increased prices for final } \\
\text { customers. }\end{array}$ \\
\hline
\end{tabular}

This way of managing the supply chain, and with the emphasis on the way of managing the transportation from the shippers to the retail market needs to change. If the system is lost, the population is lost. Losses can be evaluated in terms of millions of dollars and can be evaluated from the quality of life. The main point is: Why does the system use a lot of small trucks, an enormous amount of trucks, with bad productivity and much more pollution instead of fewer trips with bigger trucks? According to studies concerning pollution emissions done in Brazil, the USA, and several other countries, light vehicles have an average speed of $31.5 \mathrm{~km} / \mathrm{h}$ generally, but in urban areas this falls to something like $19 \mathrm{~km} / \mathrm{h}$. With this phenomenon, the emission of carbon monoxide increase by $25 \%$, hydrocarbons by $20 \%$, and fuel consumption increases by $20 \%$. The objective of this paper is to present an alternative to this scenario, using collaborative logistics substituting a lot of small trucks by fewer big trucks, decreasing costs and pollution; improving everyone's life. 


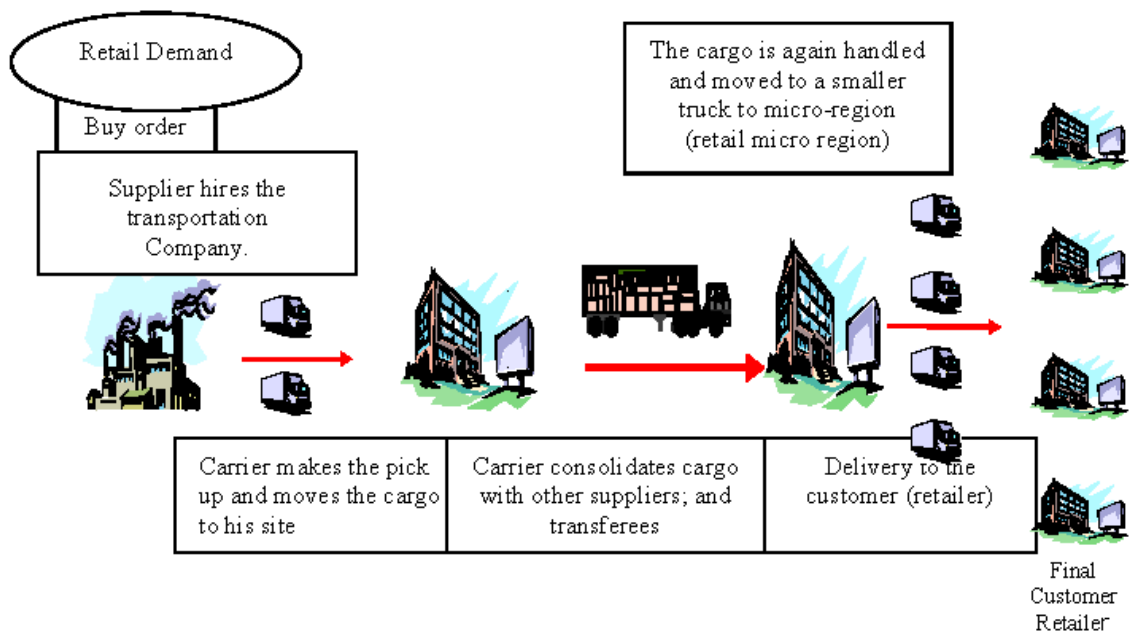

Figure 1: Schematic chart - classical delivery.

The next topic this paper will present is the study proposal of a collaborative transportation methodology for urban deliveries.

\section{Collaborative transportation concepts and the application at urban transportation}

\subsection{Collaborative transportation review}

The bibliographic review (e.g. Tacla and Potter [5]) elaborated for this research paper was broad, and besides logistics and collaborative transportation, it addressed some basic concepts related to the theme (routing, real time forwarding, mathematical modelling, dynamic transportation programming, vehicle management, etc). These themes were reviewed to give support to the study. The lack of numeric and practical implementation of collaborative transportation is one more reason to encourage the research of the theme, which is now brought to the spotlight at this stage of the paper. Collaborative Transportation (e.g. Browning and White [2]) was established as a concept in 2002, emerging from segmentation and a private study of the "CPFR" (Collaboration, Planning, Forecasting, and Replenishment). The forum generating these committees is the "VICS" (Voluntary Inter-Industry Commerce Standards Association), the American entity attempting to encourage collaboration between salespeople and buyers, through the co-management of processes and information systems. As the primary generating concept of the CTM, the CPFR has the following basic goals: Improving effectiveness; increasing sales; reducing fixed costs and working capital; reducing stock in the supply chain; increasing client satisfaction. 
In the constant search for minimizing stocks in the value chain, with shorter planning windows, transportation has become a critical matter. Nowadays transportation is a top priority for solving conflicts between buyers, sellers and transportation companies in shipping operations (processes, quantities, moments, etc.). These conflicts end up producing excessive stocks in the supply chain and under-using transportation equipment.

Management of Collaborative Transportation is an independent process. Although it occurs at the same time as the CPFR, it is established upon the same relationships between buyers and salespeople, but it incorporates new information and steps with the transportation companies. It extends the performance of the CPFR from the order confirmation, and continues up to the delivery of the product, including the commercial transactions with the transportation company, such as its payment. The opportunities for collaboration among buyers, sellers and transportation companies take place in three main categories: strategic planning, demand forecast, re-supply, and execution. CTM can therefore be summarized as: An independent process, though simultaneous to CPFR. It builds on the same relationships between buyers and sellers, incorporates new information and steps with carriers, begins at order confirmation, continues through shipment delivery, and includes carrier payment, it requires the whole process to be reengineered so that the carrier is part of the larger, more focused, buyer/seller team.

\subsubsection{The goal of CTM}

The goal of CTM is to develop collaborative relationships between buyers, sellers, transportation companies and third parties that provide logistic services (3 PLs), aiming to improve services, effectiveness and costs associated with transportation and delivery.

\subsubsection{The five stages of CTM}

For better comprehension and better scoping of the process, CTM may be divided into five stages: 1-Strategic relationships among participants (seller, buyer, transportation company); 2-Demand forecast and placement of orders; 3Orders and shared shipping process; 4-Process of freight confirmation and hiring; 5-Documentation and performance management of the process.

\subsubsection{Technological requirements}

As in any integration process, Collaborative Transportation basically depends on information in order to work properly. The generation of the necessary information and the fast and effective flow of information constitute the main technological requirements; nevertheless, there are other factors relevant to the process, such as: standardization of information; scheduling of operations; safety; openness in the project's design; management; promptness of response; transportation resources (vehicles) available for collaboration; adequate framing of information; transportation protocols (necessary formal documentation). 


\subsubsection{Critical elements for collaborative transportation}

Besides the technological requirements, there are elements in the collaboration which are critical for attaining the expected results, and which can be summarized as follows: critical mass, (which is the combination of: large freight; volumes demanded from the forwarder, shipper's capacity, sufficient resources from the provider; internet systems (Web) based on connectivity); systems via web that may reach and integrate a large group of forwarders and shippers that fosters visibility in the provision chain and that authorizes the execution of collaborative transportation; optimization technology; utilizing systematic methodology to permit encourage synergy in the net of collaborative companies to reduce the costs and improve the services.

\subsection{Urban collaborative transportation application}

Collaborative transportation, as with collaborative logistic, is hard to implement and there are few successful cases, even in countries where logistics concepts are in an advanced phase. Why is so difficult to implement collaborative logistics? The main reason: there is no collaboration in planning and interchange. Companies try to get results without planning together; it is not good enough to inform the "individual necessity" and try to conjugate it with what seems the opportunity "from the other"; even if both achieve success, this is not complete optimization or collaborative logistic if the collaboration is not complete. It is not enough to build complementary plan that attaches to the individual plan. A collaborative plan means having to build a new logistic starting from zero. Full optimization means full collaboration; collaboration means ad equating or changing individual planning to achieve the optimum for the whole collaborative group. The group can achieve an improved performance at reduced costs, but the optimum will be achieved for the system if the group works as one company as far as the collaboration effort is concerned. This paper suggests an idea of how to build a collaborative transportation system for an urban area, and specifically how to supply a Retail Company in a collaborative way. The database presented in this paper shows an important way of decreasing the fleet of delivery vehicles, and as consequence: reducing cost, pollution and saving the neighbouring population. What is the main motivation for instigating collaborative transportation in the retail supply system? The main principle is: to consolidate cargo. Several trucks of varying sizes are used to transport the cargo from the suppliers and/or from a point located at the retail region (emphasis on small and medium trucks in this case). When the system uses large numbers of trucks to transport their goods, a huge structure is necessary to cope with preparing the cargo, handling, transporting and receiving this cargo. The penalty impacts not only on the transportation cost but on the whole logistic system. The idea here is clear and simple: consolidating cargo and use fewer trucks to transport and delivery it. If the focus is technical it is really simple and easy to implement, but to become a reality, more aspects than technical focus are needed: full collaboration is necessary. It is vital to rebuild the logistic not from a shipping or supplying point of view, but from the point of view of a retailer. The system normally arranges the cargo based on deliveries per suppliers and only after that 
arranges per final customer; to optimize the system, the logic of this logistic is completely inverse. The schematics in figures 2 and 3 explain this concept. Figure 2 represents the traditional way: the retailer informs of its demand and the supplier ships goods through a carrier. Figure 3 simplifies the representation of the collaborative chain: the retailer plans the shipment together with the suppliers through a distribution center, which consolidate cargo and transport in large trucks (FTL) to the retailer.

\section{Description of the case study and development of collaborative optimization}

The case study brings together a complete retailer database regarding goods being received at a DC, to supply the stores. The retail company is a big global player and one of the three most important retailers in South America. The Distribution Center in the centre of Brazil (DCC) supplies ten stores in the region. To this application the study is considering goods that can be consolidated, which means: goods that offer no risk of being contaminated, and could be loaded together in the same truck in any situation. DCC works in this logistic chain in the traditional way, which means it demands goods directly from the suppliers, who plan the transport, hire the carriers, and ships the goods (figure 2). Using the Sao Paulo metropolitan area as the origin, all suppliers in this region are to be considered. The average delivery is 150 cases; The Sao Paulo metropolitan area represents $11.4 \%$ of all goods shipped from all regions of the country. This number permits the development of the methodology for the Sao Paulo metropolitan area without any prejudice to the chain.

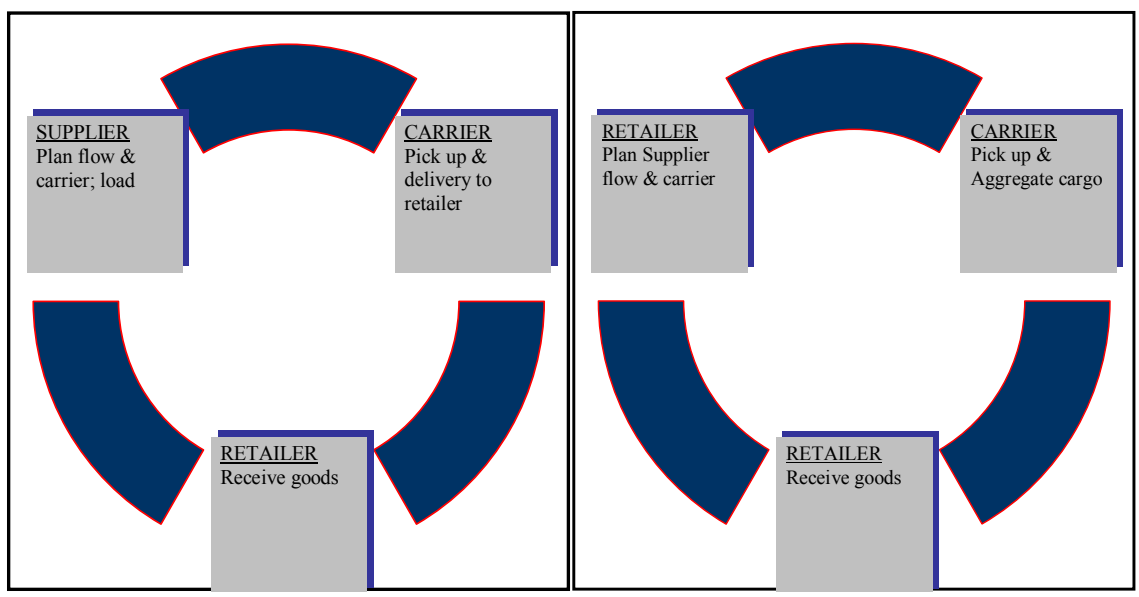

Figure 2: Traditional chain.

Figure 3: Collaborative supply retail chain. 


\subsection{Transportation costs for a real scenario}

Table 2 shows the unitary costs for each type of truck used for transportation from the suppliers in the metropolitan region to DCC. The costs pointed are based on the real market. To calculate the total transportation cost, the study uses the cases average as presented at Table 3; the criteria for that is: $80 \%$ of the cases are calculated on LLT cost, $10 \%$ of the cases are calculated on FTL cost, and $10 \%$ of the cases are calculated on small trucks. These criteria are based on the $\mathrm{ABC}$ tendency and delivery profile summarized in Table 2.

Table 2: $\quad$ Unit costs per truck type.

\begin{tabular}{|l|r|r|}
\hline \multicolumn{3}{|c|}{ Rates of Freight (US) } \\
\hline $\begin{array}{c}\text { Transport } \\
\text { Type }\end{array}$ & $\begin{array}{c}\text { Price per } \\
\text { ton }\end{array}$ & $\begin{array}{c}\text { Price per } \\
\text { case }\end{array}$ \\
\hline FTL & 61,98 & 0,62 \\
\hline LTL & 86,78 & 0,87 \\
\hline small & 153,93 & 1,54 \\
\hline
\end{tabular}

Table 3: $\quad$ Trucks per cases profile.

\begin{tabular}{|l|c|r|}
\hline \multicolumn{3}{|c|}{ Assumptions } \\
\hline $\begin{array}{c}\text { Transport } \\
\text { Type }\end{array}$ & Amount of Cases & cargo in Kg \\
\hline FTL & $>1200<2500$ & 20000 \\
\hline LTL & $>500<1200$ & 12000 \\
\hline small & $>100<500$ & 5000 \\
\hline
\end{tabular}

Table 4 brings the cost per truck type and the total cost: U\$ 51,520.55. It is important to emphasize that the database is real, but the costs are simulated.

Table 4: $\quad$ Costs simulated for real scenario.

\begin{tabular}{|c|c|c|}
\hline Truck Type & \# Cases & Cost \\
\hline LTL & 45286 & $39.298,12$ \\
\hline FTL & 5661 & $3.508,76$ \\
\hline small & 5661 & $8.713,67$ \\
\hline & 56608 & $51.520,55$ \\
\hline
\end{tabular}

\subsection{Transportation costs for a collaboration scenario}

Using the premises for the database and the criterion as explained before, the cargo shipped to DCC from Sao Paulo metropolitan region was consolidated as presented in Table 5. 
Table 5: Cargo consolidated trough collaboration.

\begin{tabular}{|c|c|c|c|c|c|c|c|c|c|c|}
\hline date & \# received & \# invoice & max case & $\begin{array}{c}\text { average case } \\
\text { per invoice }\end{array}$ & $\begin{array}{l}\text { DesvPad } \\
\text { case }\end{array}$ & total case & FTL & LTL & small & courrier \\
\hline 20060106 & 38 & 38 & 160 & \begin{tabular}{r|}
39 \\
\end{tabular} & 44 & 1476 & 1 & \begin{tabular}{|l|}
0 \\
\end{tabular} & 0 & 0 \\
\hline 20060109 & 98 & 98 & 592 & 29 & 73 & 2801 & 1 & 0 & 1 & 0 \\
\hline 20060110 & 44 & 44 & 264 & 17 & 40 & 768 & 0 & 1 & 0 & 0 \\
\hline 20060111 & 148 & 148 & 193 & 15 & 24 & 2184 & 1 & 0 & 0 & 0 \\
\hline 20060112 & 215 & 215 & 408 & 20 & 53 & 4252 & 2 & 0 & 0 & 0 \\
\hline 20060113 & 51 & 51 & 195 & 22 & 31 & 1101 & 1 & 0 & 0 & 0 \\
\hline 20060116 & 257 & 257 & 269 & 13 & 32 & 3450 & 2 & 0 & 0 & 0 \\
\hline 20060117 & 181 & 181 & 320 & 15 & 36 & 2730 & 2 & 0 & 0 & 0 \\
\hline 20060118 & 139 & 139 & 360 & 27 & 51 & 3685 & 2 & 0 & 0 & 0 \\
\hline 20060119 & 40 & 40 & 140 & 23 & 22 & 931 & 0 & 1 & 0 & 0 \\
\hline 20060120 & 94 & 94 & 320 & 34 & 51 & 3182 & 2 & 0 & 0 & 0 \\
\hline 20060121 & 3 & 3 & 380 & 260 & 107 & 780 & 0 & 1 & 0 & 0 \\
\hline 20060123 & 149 & 149 & 296 & 14 & 33 & 2053 & 1 & 0 & 0 & 0 \\
\hline 20060124 & 237 & 237 & 679 & 20 & 55 & 4771 & 2 & 0 & 0 & 0 \\
\hline 20060125 & 122 & 122 & 168 & 17 & 31 & 2134 & 1 & 1 & 0 & 0 \\
\hline 20060126 & 60 & 60 & 170 & 25 & 29 & 1494 & 1 & 0 & 1 & 0 \\
\hline 20060127 & 46 & 46 & 320 & 25 & 46 & 1168 & 0 & 1 & 0 & 0 \\
\hline 20060130 & 83 & 83 & 340 & 27 & 48 & 2266 & 1 & 0 & 0 & 0 \\
\hline 20060131 & 237 & 237 & 132 & 10 & 17 & 2335 & 1 & 0 & 0 & 0 \\
\hline 20060201 & 71 & 71 & 384 & 33 & 69 & 2337 & 1 & 0 & 0 & 0 \\
\hline 20060202 & 145 & 145 & 480 & 39 & 69 & 5597 & 3 & 0 & 0 & 0 \\
\hline 20060203 & 110 & 110 & 192 & 20 & 28 & 2184 & 1 & 1 & 0 & 0 \\
\hline 20060206 & 47 & 47 & 165 & 31 & 38 & 1437 & 1 & 0 & 0 & 0 \\
\hline 20060207 & 39 & 39 & 384 & 38 & 73 & 1492 & 1 & 0 & 0 & 0 \\
\hline Total & 2654 & 2654 & 679 & 21 & 46 & 56608 & 28 & 6 & 2 & 0 \\
\hline
\end{tabular}

This optimization is relevant, for the real scenario, 262 trucks were used and for the study scenario, 36 trucks were calculated. The transportation costs from the DC in Sao Paulo metropolitan area to the DCC is U\$ 39,271.02, however to calculate the total cost is necessary to simulate the milk run cost to DC in Sao Paulo metropolitan area and the handling costs in the DC. Those costs are presented in Table 6 and the costs are based on the market rates.

Table 6: $\quad \mathrm{a}$ and $\mathrm{b}$ : total cost.

\begin{tabular}{|c|c|c|c|}
\hline $\begin{array}{c}\text { Transport } \\
\text { Type }\end{array}$ & Transfer cost & $\begin{array}{c}\text { Cost per } \\
\text { case }\end{array}$ & Total Cost \\
\hline FTL & $31.239,67$ & & 509472 \\
\hline LTL & $5.206,61$ & Ml11k Run & $\frac{0.094,12}{464186}$ \\
\hline small & $2.770,74$ & DC handle & $4.641,86$ \\
\hline & $39.217,02$ & & $9.736,58$ \\
\hline
\end{tabular}

Total cost of the optimized scenario, after application the collaboration methodology is: U\$ 48,953.60; the directly saving is 5\%.

\section{Conclusions}

If fully applied to all products and all regions, the methodology will produce an outcome even more relevant. The integration of the strategic and tactic hierarchical levels with the operations used in the simulation allows for the 
construction of a scenario more favourable to route conjugation, and, therefore, savings in freight hiring. The results, however, are already significant. It becomes clear that the proposal is effective when conjugated with the complete methodology. This way the goals are met, to the extent that a quantitative implementation of the concepts in collaborative transportation becomes material, with added relevance of a case of the transportation of large volumes within large geographical areas. The indirect results (the non-financial advantages) are maybe of even greater importance:

- Savings in handling at DCC; savings in unloading resources;

- Savings in truck fixed costs, with lower time in lines to unload trucks;

- Direct environmental benefits, with fewer large trucks emitting less pollution than many small trucks;

- Direct benefits for the population, with fewer trucks meaning less noise and less traffic.

Comparing the initial numbers of trucks that arrived at the DCC with the number of trucks after the methodology application, the result is huge: 262 vs. 36 . As a result, 226 trucks, will not arrive at the DCC in a database period. The 226 trucks represent a reduction of $86.2 \%$. If the collaborative transportation concepts could be implemented on a large scale, the results would be fantastic for the logistic system and for society. The case study only referred to one retailer DC, and for one type of product from one region. Collaborative logistics and collaborative transportation are necessary not only to reduce cost, but also to save natural resources, the environmental and the population in the future.

\section{References}

[1] Bowersox D. J., Closs D. J. Logistical Management: The Integrated Supply Process. McGraw-Hill. USA, 1996.

[2] Browning, B. White, A. Collaborative Transportation Management - A Proposal, USA, 2001.

[3] Desrochers, J. Soumis, F; Belanger, N; Ioachim. Fleet Assignment and Routing With Schedule Synchronization Constraints. I. European Journal of Operation Research 119 (Elsevier Science), 1999.

[4] Lima Jr., O. F. A Carga na Cidade: Hoje e Amanhã, LAL- Universidade Estadual de Campinas, 2005.

[5] Tacla, D., Botter, R. Study of Collaboration Transportation of large volumes of bulk cargoes, with application in case of soy and fertilizers. White paper, 2003. 